\title{
Consideraciones para el Rediseño de la Línea de Ensamble de Vehículos Aéreos No Tripulados desarrollados por la Fuerza Aérea Ecuatoriana*
}

\section{Considerations of the Redesign for the Assembly Line of Unmanned Aircraft Vehicles Developed by the Ecuadorian Air Force}

\author{
DOI: http://dx.doi.org/10.17981/ingecuc.12.1.2016.05
}

Artículo de investigación - Fecha de recepción: 30 de Septiembre de 2015 - Fecha de aceptación: 2 de Diciembre de 2015

\section{Ricardo Daniel Fiallos Castillo}

Ingeniero Industrial, Universidad Técnica de Ambato. Ambato (Ecuador). fiallosricardo@yahoo.es

John Paúl Reyes Vásquez

Magíster en Ingeniería Industrial, Universidad Técnica de Ambato. Ambato (Ecuador). johnpreyes@uta.edu.ec

Paúl Homero Armas Ramírez

Mayor del Ejército, Centro de Investigación y Desarrollo de la Fuerza Aérea Ecuatoriana (CIDFAE), Ambato (Ecuador). paul.armas@cidfae.gob.ec

Mario Geovanny García Carrillo

Magíster en Redes y Telecomunicaciones, Universidad Técnica de Ambato. Ambato (Ecuador). marioggarcia@uta.edu.ec

Para citar este artículo / To reference this paper:

R. D. Fiallos Castillo, J. P. Reyes Vásquez, P. H. Armas Ramírez y M. G. García Carrillo "Consideraciones para el ReDiseño de la Línea de Ensamble de Vehículos Aéreos No Tripulados Desarrollados por la Fuerza Aérea Ecuatoriana", INGE CUC, vol. 12, no. 1, pp. 51-64, 2016. DOI: http://dx.doi.org/10.17981/ingecuc.12.1.2016.05

Resumen-- Este trabajo tiene como objetivo el re-diseño de la línea de ensamblaje de los vehículos aéreos no tripulados (UAV, por sus siglas en inglés) destinados a realizar diversas misiones a grandes alturas llevadas a cabo en el Centro de Investigación y Desarrollo de la Fuerza Aérea Ecuatoriana (CIDFAE). La metodología que se aplicó consta de tres fases: la primera comprende el análisis de la situación estratégica actual; la segunda estima la capacidad del proceso productivo con el uso de las normas de tiempo predeterminadas con base en el sistema MTM-2; la tercera realiza el estudio de la distribución de las instalaciones mediante simulación. Como resultado final se opta por una distribución mixta que garantiza una producción en serie de UAV. La construcción de piezas se realiza en células de fabricación y el ensamble final por estaciones de trabajo; además, se establecen estrategias para el fortalecimiento de la capacidad operativa de producción de la instalación que se proyecta para tres UAV producidos cada dos meses.

Palabras claves-- Producción; UAV; estrategia de operaciones; normas de tiempos predeterminadas; MTM-2; balanceo de línea de ensamble; distribución de instalaciones; simulación del proceso productivo.

\begin{abstract}
This paper presents the redesign of an assembly line in the manufacturing of UAVs used in various missions that take place at high altitudes for the Research and Development Center of the Ecuadorian Air Force (CIDFAE). The methodology that was applied consists of three phases: the first one involves the analysis of the current strategic situation; the second one estimates the capacity of production, time standards based on the MTM-2 system; and the third one performs a distribution study of the plant facilities through simulation. As result a mixed distribution is chosen since it guarantees a production batch of UAVs. The parts are produced in manufacturing cells and the final assembly in workstations. Furthermore, strategies for strengthening the operational capacity of the plant will be established in order to manufacture three UAVs every two months.
\end{abstract}

Keywords-- Production; UAV; Operations Strategy; Standards of Predetermined Times; MTM-2; Assembly Line Balancing; Distribution Facilities; Simulation of the Production Process.

\footnotetext{
* Artículo de investigación científica derivado del proyecto de investigación titulado, "Assembly process drones for Research and Development Center of the Ecuadorian Air Force (CIDFAE)". Financiado por la Universidad Técnica de Ambato y el Centro de Investigación y Desarrollo de la Fuerza Aérea Ecuatoriana (CIDFAE). Fecha de inicio: agosto de 2014. Fecha de finalización: agosto de 2015.
} 


\section{INTRODUCCIÓN}

En los últimos años, los vehículos aéreos no tripulados han demostrado gran versatilidad en diferentes aplicaciones que van desde el monitoreo $\mathrm{y}$ vigilancia hasta labores de rescate y reconocimiento militar, ahorrando costos y reduciendo el riesgo de pérdidas humanas en operativos militares y civiles. Debido a su reciente aparición hay escasez de datos estadísticos relacionados con el diseño de estos sistemas [1], por lo cual no existen metodologías de diseño y los fabricantes actuales deben involucrarse en mayor grado en el área de investigación.

En Latinoamérica el desarrollo tecnológico de los vehículos aéreos no tripulados (UAV, por sus siglas en inglés) no representa una gran actividad económica, sin embargo, se está incrementando notablemente con el paso de los años. En la región existen varios países muy activos en programas de desarrollo y utilización de UAV, entre ellos están: Brasil, México, Argentina, Colombia, Ecuador, Chile, Uruguay y Venezuela [2]. En Ecuador, el Centro de Investigación de la Fuerza Aérea Ecuatoriana (CIDFAE) se ha convertido en un referente en el proceso de investigación, desarrollo e innovación de plataformas aéreas no tripuladas. Actualmente, se encuentra inmerso en la producción de una serie corta de aeronaves no tripuladas denominada Proyecto Gavilán III [3] para contribuir al cambio de la matriz productiva nacional. Los constantes cambios y requerimientos que surgen por parte de los consumidores orientan a los fabricantes a incorporar en su sistema de gestión, ciclos de mejora continua para mantenerse dentro del mercado, por lo cual para el caso de las industrias manufactureras, las líneas de producción también poseen un tiempo de vida útil y necesitan renovarse continuamente o generar un nuevo modelo para el proceso productivo.

Toda empresa diseña planes estratégicos para el logro de sus objetivos planteados, esto implica el desarrollo de una metodología con un proceso cuidadoso de toma de decisiones [4], [5]. La planeación estratégica tiene dos funciones principales dentro de las organizaciones ya que se encarga de desarrollar, integrar y coordinar un plan de acción consistente, y además, facilitar la adaptación a los cambios del entorno [6]; para ello requiere de un proceso básico en el cual se identifican los distintos elementos que representan la razón de ser de una institución, como la misión, visión y objetivos de la ésta.

Solamente un modelo de gestión estructurado junto con la integración de las unidades que componen la empresa pueden garantizar el direccionamiento de las funciones misionales de una organización hacia la competitividad [7], [8]. El éxito de una organización depende de la forma en que ésta responde a los cambios que se presentan y la metodología que desarrolla para adaptarse a los mismos. Las cinco fuerzas de Porter permiten analizar todos los aspectos favorables y desfavorables del sistema dinámico en el cual la empresa compite. El modelo se basa en dos tipos de enfoque: (a) cuando una empresa se encuentra fuera del negocio y quiere ingresar a él y (b) cuando una empresa se encuentra dentro del negocio y otras empresas competidoras quieren entrar a él.

Teniendo en cuenta estos aspectos es posible desarrollar estrategias de operaciones que incluyan decisiones, como los nuevos productos que deben desarrollarse y cuándo deben introducirse en la producción, la necesidad de nuevas instalaciones y los esquemas de producción a seguir de los nuevos productos y servicios [9]. El control de estos procesos debe convertirse en parte fundamental de los objetivos de calidad de las organizaciones [10] para que de esta manera las mismas puedan ofertar productos y servicios de calidad al mercado.

Para que el CIDFAE oferte productos de calidad se requiere de un proceso productivo que utilice los recursos de manera eficiente para reducir costos de fabricación, por esta razón surge la necesidad de llevar a cabo un estudio especializado para identificar posibles falencias y fortalecer la capacidad operativa de la industria. Entre las técnicas utilizadas para el estudio de los procesos operativos se encuentran el estudio de métodos, el estudio de tiempos por cronómetro y otras técnicas muy poco utilizadas, como las normas predeterminadas (NTPD). Estos sistemas se desarrollan a partir de los valores asignados a las operaciones corporales (micromovimientos) que el analista selecciona de un manual después de analizar el método de trabajo y los suma para obtener el tiempo necesario para efectuar un determinado trabajo [11]. Se estima que actualmente existen alrededor de 50 sistemas diferentes de medición de este tipo, de los cuales los sistemas más utilizados a nivel mundial son el sistema MTM (Methods Time Measurement) y Work Factor.

Estos sistemas pueden ser utilizados solamente cuando no se dispone de otra forma de medir el tiempo de ciclo de un determinado proceso, es decir, cuando se va a realizar el diseño de una nueva línea de producción, o cuando se requiere idear una nueva metodología de trabajo [12].

Por otra parte, además de acondicionar los procesos de acuerdo con las necesidades de producción es necesario contar con una infraestructura adecuada que esté en consonancia con el producto que se desea fabricar, por esta razón una distribución de instalaciones idónea se debe integrar de la mejor manera a los elementos que intervienen en el proceso para poder garantizar un flujo continuo de los materiales y una distancia mínima entre las operaciones que se lleven a cabo [13], [14]. Una dis- 
tribución de planta óptima busca resolver los problemas de diseño que incluye la partición de una región plana en departamentos o superficies de trabajo con el fin de minimizar los costos asociados con interacciones proyectadas entre los departamentos [15], [16], de esta manera se utiliza de forma eficiente el espacio disponible y se ofrece satisfacción y seguridad al trabajador.

Con el advenimiento de los nuevos sistemas de información, rápidamente los sistemas tradicionales de manufactura están volviéndose obsoletos y con ello aparece una nueva generación de instalaciones con sistemas de manufactura celular. Este giro reciente hacia este tipo de sistemas necesita del rediseño de la planta y su distribución. Las distribuciones por producto y proceso tradicionales se están reemplazando por aquellas distribuciones de tipo herradura, conformadas por células de trabajo pequeñas y semicirculares [17]. La aplicación de software mediante el cual se efectúa una visualización del comportamiento de procesos reales en un contexto virtual, junto con el enfoque de gestión por procesos, se consideran herramientas fundamentales que se complementan entre sí para el análisis del desempeño de las operaciones y, por ende, son base fundamental para la mejora continua del proceso productivo [18] [19].

Para una producción eficiente dentro de una industria se debe también considerar la gestión de la materia prima a lo largo de la cadena de suministro. Si bien es cierto que los modelos tradicionales de inventarios proveen una solución, es necesario entender qué variables deben mantenerse y cuáles deben cambiarse [20]. El modelo de gestión de dinámica de amortiguadores, suministra materiales utilizando la teoría de restricciones y asegura el nivel de inventario objetivo en el tiempo eficientemente [21].

Se considera importante la inclusión de planes agregados de producción basados en algoritmos de programación lineal, ya que optimizan los recursos de manera sistemática. De esta manera, se puede tomar una decisión más adecuada para llevar a cabo la producción dentro de las empresas manufactureras en general [22].

Otro aspecto trascendental es la aplicación de un análisis costo/beneficio mediante el cual se pueda identificar el impacto de las propuestas o alternativas de solución a un problema propuesto, pues con ello se logra minimizar defectos, evitar tiempos muertos, estandarizar procesos e incrementar la productividad de una empresa [23].

Estos conceptos pueden aplicarse a diversos sectores productivos dentro del país, entre estos, el diseño y la fabricación de prototipos de UAV. Para involucrarse en el desarrollo de una serie corta de aeronaves no tripuladas, sin dejar el proceso de investigación y desarrollo, el CIDFAE pretende modificar los procesos actuales incorporando el concepto de escalamiento industrial, el mismo que consiste en la adquisición de capacidades tecnológicas y vínculos de mercado que permita mejorar su competitividad y moverse hacia actividades de mayor valor, todo ello en el marco de la innovación. Además, se busca incorporar nuevas capacidades tecnológicas, por medio del desarrollo de un verdadero clúster aeronáutico, cuyo objetivo es el diseño de nuevas metodologías y procedimientos para la mejora de la gestión táctica y a tiempo real de las operaciones de las nuevas aeronaves ecuatorianas.

Para cumplir con el propósito planteado se establece un análisis estratégico mediante el cual se identifican los factores internos y externos que influyen positiva y negativamente en el desarrollo de este tipo de productos. Consecutivamente, se evalúa el proceso productivo con herramientas para el análisis de procesos y se plantea la solución.

Gracias a este estudio también se da a conocer la aplicación de las normas de tiempo predeterminadas en un caso práctico, sobre el cual no se ha observado registro de estudios anteriores a nivel nacional. Producto de ello, se desarrolla el análisis de la distribución de las instalaciones con la aplicación de un software para acondicionar el hangar. Como resultado final se obtendrá una distribución que dará cuenta de un diseño del área que esté de acuerdo con las necesidades de producción, que para el ensamblaje final tendrá un gran impacto social y científico.

El presente trabajo se fundamenta en la necesidad del rediseño de la línea de ensamblaje de aviones no tripulados que actualmente desarrolla la CIDFAE, partiendo de la necesidad de un proceso estandarizado para desarrollar las operaciones de manera eficiente y que a su vez permita la fabricación en serie de este artefacto.

El trabajo se ha estructurado en cinco secciones de la siguiente manera: la introducción sitúa el problema, describe los antecedentes teóricos y la estrategia de investigación; la metodología presenta el enfoque y método de investigación; los resultados contribuyen a la solución del problema, se realiza una discusión y se presentan las conclusiones.

\section{Metodología}

El estudio se realiza con una investigación de campo utilizando técnicas de observación directa y recolección de información con base en entrevistas y formularios. La información recolectada recibe el tratamiento adecuado basado en una fundamentación teórica obtenida mediante una investigación bibliográfica previamente establecida. El desarrollo del proyecto comprende tres fases importantes: la primera se refiere al análisis estratégico de la situación actual del CIDFAE, en el cual se identifican los elementos de la planeación estratégica, luego se realiza el análisis del sector industrial 
mediante el modelo de las cinco fuerzas de Porter, y por último con base en un análisis interno y externo se plantean estrategias de producción con las cuales se busca mejorar la calidad de los procesos $\mathrm{y}$ productos que se desarrollan[24]; la segunda, corresponde al estudio del proceso productivo que identifica cada uno de los componentes y el proceso de fabricación, el análisis se complementa con un estudio de tiempos utilizando las normas de tiempo predeterminadas del sistema MTM. Con los datos obtenidos se calcula la capacidad de producción y se identifican los procesos requeridos para efectuar el ensamble final. Mediante un balance de la línea de ensamble final, en la tercera y última fase, se desarrolla un diseño para la distribución de planta que facilite la producción en serie de los UAV tipo Gavilán III.

Dentro del sistema MTM existen las bases de datos MTM-1, MTM-2 y MTM-3 que guardan relación directa entre sí. El sistema MTM-1 es mucho más preciso que el MTM-2, ya que el primero presenta una base de datos completa, mientras que el segundo se basa en una combinación de micromovimientos presentes en el MTM-1 [25]. A pesar de ello, el sistema MTM-2 arroja resultados muy aproximados y se convierte en una herramienta fundamental para el análisis de ciclos con tareas poco repetitivas y tiempos muy prolongados. Este sistema de tiempos predeterminados es el más utilizado en la industria. Cada micromovimiento está determinado en unidades de medición de tiempo (TMU), donde un TMU equivale a 0.0006 minutos o lo que es igual a 0.036 segundos [26]. En general, el sistema MTM-2 se recomienda aplicarlo bajo condiciones de trabajo en donde la parte de esfuerzo del ciclo de trabajo es de más de un minuto de duración, el ciclo no es altamente repetitivo y la parte manual del ciclo de trabajo no implica un gran número de movimientos manuales complejos o simultáneos.

Para este trabajo se utiliza esta metodología de estudio de tiempos con NTPD apoyados en el sistema MTM-2 por dos grandes razones, la primera: la CIDFAE se dedica únicamente a la investigación y desarrollo de diferentes UAV, por lo cual no existen registros de estudios del trabajo que se hayan desarrollado anteriormente, y la segunda es que al tratarse de un modelo nuevo, y que además se requiere fabricar en serie, es necesario proyectar el sistema productivo, los movimientos que realizan los operarios para realizar los diferentes trabajos y la maquinaria existente en la sección. Aunque esta metodología no es una técnica de medición directa, ofrece resultados muy aproximados a la realidad ya que son fruto de múltiples estudios desarrollados en diferentes áreas y que además son avalados por la Organización Internacional del Trabajo (OIT). Así, con los datos obtenidos de este análisis se estima una capacidad de producción futura de la instalación.

\section{III.RESULTADOS}

\section{Fase I: Diagnóstico estratégico de la institución}

\section{A. Identidad corporativa}

El Centro de Investigación y Desarrollo de la Fuerza Aérea Ecuatoriana tiene como misión desarrollar investigación e innovación científica y tecnológica aeroespacial para mejorar las capacidades operativas de la Fuerza Aérea, apoyar al desarrollo nacional y disminuir la dependencia tecnológica del extranjero, puesto que pretende ser una Fuerza Aérea disuasiva, respetada y aceptada por la sociedad y pionera en el desarrollo aeroespacial nacional. Para ello reúne valores y principios que son el cimiento de esta noble institución y que ejercen singular influencia en el desarrollo de las diferentes actividades de sus colaboradores. Su estructura organizacional está conformada por tres niveles jerárquicos: nivel directivo, que son los responsables de formular planes y programas para la institución; nivel auxiliar, que se encarga de la situación financiera; y el nivel operativo, que se encarga de la producción y transferencia de tecnología.

\section{B. Análisis del sector industrial}

De acuerdo con el enfoque de las cinco fuerzas de Porter, se considera que el CIDFAE se encuentra fuera de la industria de producción masiva de $\mathrm{UAV}$, ya que los modelos desarrollados hasta el momento fueron realizados simplemente con fines investigativos.

En América Latina, la fabricación de UAV empieza a expandirse con perspectivas sumamente ambiciosas gracias a la inversión de los gobiernos y otras instituciones en el desarrollo científico y tecnológico. En esta misma región, los países que lideran esta industria son Brasil y Argentina, lo cuales han desarrollado una variedad de modelos para uso militar y civil; además, países como México, Colombia, Ecuador, Chile, Uruguay y Venezuela también han incursionado en esta área en menor grado, sin embargo, todos éstos pueden considerarse como amenaza de nuevos competidores. Dentro de estas naciones existen varias empresas y centros de investigación y desarrollo aeronáutico, por lo cual el nivel de competencia en esta industria es sumamente alto, como se puede apreciar en la Tabla I.

Los drones desarrollados a nivel local están destinados a tareas de investigación, monitoreo y búsqueda de información, por lo cual dentro del país son muy codiciados por empresas como Petroecuador y otras instituciones. El poder de negociación de los clientes se considera medio ya que se requiere mayor inversión para proveer productos de última generación al mercado local. 
Tabla I. Fabricantes de UAV en Latinoamérica

\begin{tabular}{|c|c|}
\hline \multicolumn{2}{|r|}{ Fabricantes de UAV en Latinoamérica } \\
\hline Argentina & $\begin{array}{l}\text { - Fábrica Argetnian de Aviones (FAdeA) } \\
\text { - Florestan } \\
\text { - Tesacom } \\
\text { - Volartec } \\
\text { - Nostromo } \\
\text { - Fixvieuw } \\
\text { - Tedimec } \\
\text { - Aerodreams } \\
\text { - Instituto Aeronáutico Universitario (IAU) } \\
\text { - Universidad Tecnológica Nacional (UTN) } \\
\text { - INVAP }\end{array}$ \\
\hline Brasil & $\begin{array}{l}\text { - Comando General de Tecnología Aeroespacial } \\
\text { (CTA) } \\
\text { - Flight Technologies } \\
\text { - Avibras } \\
\text { - Easystech } \\
\text { - Santos lab } \\
\text { - AGX Tecnología } \\
\text { - Embravant } \\
\text { - Incubaero } \\
\text { - Xmobots } \\
\text { - Universidad de Minas Gerais } \\
\text { - Instituto de Investigación y Desarrollo } \\
\text { - Instituto de Aeronáutica y Espacio }\end{array}$ \\
\hline Colombia & $\begin{array}{l}\text { - EFIGENIA AEROSPACE } \\
\text { - Corporación de la Industria Aeronáutica Co- } \\
\text { lombiana } \\
\text { - Universidad de San Buenaventura }\end{array}$ \\
\hline Chile & $\begin{array}{l}\text { - IDETEC } \\
\text { - Industrias Eléctricas RMS } \\
\text { - Academia Politécnica Aeronáutica } \\
\text { - Ejército de Chile }\end{array}$ \\
\hline Perú & $\begin{array}{l}\text { - Centro de Desarrollo de Proyectos (CEDEP) de } \\
\text { la Fuerza Aérea Peruana } \\
\text { - Armada peruana }\end{array}$ \\
\hline Uruguay & - Fuerza aérea uruguaya \\
\hline Venezuela & $\begin{array}{l}\text { - Carabay } \\
\text { - Compañía Anónima Venezolana de Industrias } \\
\text { Militares } \\
\text { - Aviación Militar Bolivariana de Venezuela } \\
\text { - Universidad Simón Bolívar } \\
\end{array}$ \\
\hline México & $\begin{array}{l}\text { - HYDRA TECHNOLOGIES } \\
\text { - Instituto de Investigación y Desarrollo de la } \\
\text { Secretaría de Marina SEMAR }\end{array}$ \\
\hline Ecuador & $\begin{array}{l}\text { - Centro de Investigación y Desarrollo de la } \\
\text { Fuerza Aérea Ecuatoriana CIDFAE }\end{array}$ \\
\hline
\end{tabular}

Fuente: [2].
La amenaza de productos sustitutos tiene un grado de incidencia bajo ya que estos productos no son fácilmente reemplazados por otros sistemas debido a las prestaciones físicas y técnicas que presentan, como tiempo de vuelo, capacidad de carga útil, entre otros aspectos técnicos.

En cuanto al poder de negociación de los proveedores se considera que el nivel de incidencia es bajo debido a que existe gran cantidad de proveedores a nivel nacional. Los precios no difieren en gran medida, por lo cual la institución tiene la posibilidad de elegir e incluso imponer en parte sus condiciones.

Actualmente, se concluye que la rivalidad entre competidores dentro del país es nula, pero a nivel de Latinoamérica esta industria se encuentra en activo crecimiento. Por esta razón, se considera importante adoptar nuevas medidas y estrategias que permitan al CIDFAE adaptarse a los constantes cambios tecnológicos y del mercado.

\section{Diagnóstico estratégico del Centro de Investigación y Desarrollo de la Fuerza Aérea Ecuatoriana (CIDFAE)}

La formulación y direccionamiento estratégico parten de la situación actual de la institución, por esta razón es indispensable la correcta obtención, análisis y procesamiento de los factores internos (fortalezas y debilidades) y factores externos (oportunidades y amenazas).

Las principales fortalezas de la institución son que cuenta con un sólido proceso de investigación, desarrollo e innovación continua; posee fuerza laboral con estabilidad laboral; existe la infraestructura, maquinaria y equipos necesarios para las respectivas pruebas; además, el producto que se oferta es de alta calidad, hecho por manos ecuatorianas y único en el mercado nacional.

Entre las debilidades se tienen la inexistencia de procesos de fabricación estandarizados; la institución se encuentra fuera del mercado de producción masiva de UAV; además existe innovación tecnológica limitada; y además se tiene la necesidad de capacitación al personal.

Entre las oportunidades, se destaca el apoyo del gobierno a la producción nacional; la institución se encuentra en capacidad de desarrollar equipo tecnológico nacional; y existe interés en el proyecto por parte de grandes empresas e instituciones. En cuanto a materiales, existe gran variedad de proveedores a nivel local y nacional.

Entre las posibles amenazas se destaca la importación de UAV de última tecnología; además, existe un alto grado de crecimiento e innovación en esta área por parte de varios países a nivel de Latinoamérica. También, las políticas de importación ocasionan elevados costos de materiales, equipos y herramientas que se requieren para el proceso productivo. 
Para lograr posicionarse en el mercado es necesario concentrarse en el cliente y sus requerimientos, luego se debe definir la estrategia más apropiada que determine el programa de acción a seguir, y por último, hay que seleccionar la estrategia más apropiada posicionándose entre las tres opciones: mejor producto, solución total del cliente y el sistema Lock- In, es decir, un enfoque más allá de nuestra industria más cercana [27].

\section{Planteamiento de estrategias para mejorar la capacidad operativa del CIDFAE}

En este literal se describen algunas de las estrategias con las cuales se pretende mejorar la capacidad operativa del CIDFAE.

Es de gran importancia la inserción de los UAV a diferentes aplicaciones y sectores productivos, además, es necesario que la institución adopte planes de mejora continua mediante el apoyo de entidades externas para elevar la capacidad de producción y distribución del producto. Se debe explotar la capacidad operativa para desarrollar nuevos productos y servicios, establecer convenios con grandes empresas para fomentar el desarrollo tecnológico y adaptar los productos con base en las necesidades.

Para enfrentar las debilidades y aprovechar las oportunidades es esencial fomentar la integración entre departamentos del CIDFAE por medio de capacitaciones continuas. Se deben estandarizar los procesos operativos con el fin de ofrecer productos de calidad al más bajo costo, establecer convenios con nuevos proveedores, buscar nuevos productos y materia prima al más bajo costo y establecer planes de mantenimiento preventivo para maquinaria y equipos.

Entre las estrategias para maximizar las fortalezas y minimizar las amenazas se propone el lanzamiento de productos innovadores a bajo costo aplicables a distintos sectores productivos, el desarrollo de planes de contingencia relacionados con cambios en leyes gubernamentales o inestabilidad financiera, la adecuación de la infraestructura existente a las necesidades de producción, optimizar los recursos asignados por parte del Gobierno, la adquisición de tecnología de última generación para crear nuevos y mejorados prototipos y establecer alianzas estratégicas con países productores de tecnología a nivel local. Asimismo, como pilar fundamental, es importante fomentar el estudio y la medición del trabajo en el proceso productivo de fabricación y ensamble de UAV, y por último, implantar nuevos sistemas de manufactura para reducir el desperdicio de materiales y optimizar recursos.

\section{Fase II: Estudio del proceso productivo}

En este apartado se analiza de manera general los procesos de fabricación requeridos para el desarrollo de los UAV tipo Gavilán III.
Existen doce pasos dentro del proceso de diseño de una aeronave en general, para el caso de los sistemas aéreos no tripulados (UAV) no existe ninguna excepción. El proceso a seguir es el siguiente:

Requerimientos del solicitante: son las capacidades que debe cumplir el prototipo desarrollado para la aceptación por parte del usuario.

Pensamiento de diseño: aquí se estudian todos los parámetros referentes al propósito para el cual será destinado el prototipo, la factibilidad de su construcción, los diseños con conceptos aerodinámicos y la tecnología a emplearse, entre otros.

Modelamiento geométrico: una vez que se ha definido un posible diseño con parámetros definidos, se procede a graficar el prototipo en un software CAD.

Análisis aerodinámico: el cual se efectúa con base en cálculos hechos a mano con varias ecuaciones y la aplicación de software especializado para la obtención de ciertas ecuaciones que faciliten el diseño. En la Fig.1 se muestra la simulación y análisis aerodinámico de un prototipo UAV mediante el programa SolidWorks.

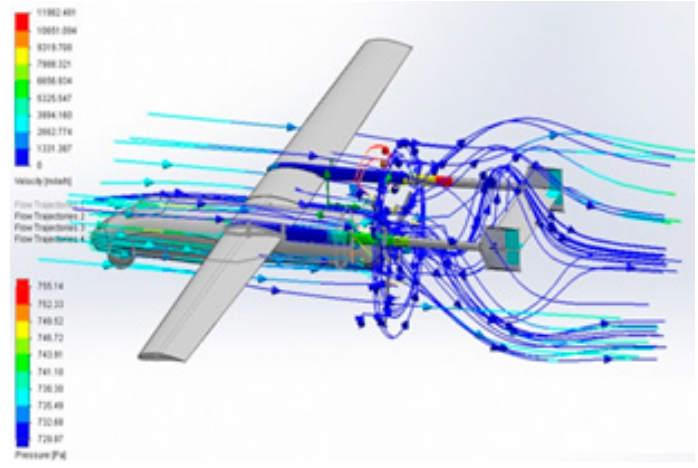

Fig. 1. Simulación aerodinámica del prototipo UAV. Fuente: [28].

Modelamiento propulsivo: está relacionado con la selección del motor que el prototipo va a utilizar para cumplir con su misión específica de la manera más eficiente.

Análisis de restricciones: tiene que ver con las limitantes del prototipo en vuelo. En esta fase se analiza la velocidad máxima que va a poder alcanzar el prototipo, distancias de despegue y aterrizaje y otros parámetros necesarios para alcanzar un equilibrio en el prototipo $\mathrm{y}$ que él mismo pueda cumplir con la función encomendada.

Análisis de misión: se enfoca en las fases de vuelo que el prototipo va a cumplir. Una típica misión de un avión no tripulado sería despegue, aceleración, ascenso, crucero, reconocimiento, movimiento a objetivo, dispersión, crucero, aterrizaje. 
Análisis de pesos de componentes: es fundamental para determinar el centro de gravedad del avión y sus márgenes operativos. En este paso se ingresan los datos de los pesos de todos los equipos que van a ir en el prototipo.

Análisis de tamaño: es el paso en el que el diseñador ubica todos los equipos de vuelo dentro de la aeronave y reduce el espacio físico del fuselaje para que el avión sea más eficiente en pleno vuelo.

Análisis de costo: se determina el valor estimado de inversión en el desarrollo del prototipo y la posterior producción.

Optimización: se efectúan simulaciones en software especializado para modificar la forma de las alas de tal manera que reduzca la resistencia al aire a su mínima expresión posible para reducir costos en el consumo de combustible.

Reporte de rendimiento: está ligado directamente con pruebas experimentales del prototipo, ya sea en túneles de viento o propiamente construyendo el prototipo y posteriormente realizando las pruebas de vuelo necesarias [28].

El UAV Gavilán III, al igual que varios modelos de este tipo, se compone de ciertos sub-ensambles principales, como los que se muestran en la Fig. 2. Para la fabricación de estos prototipos, la materia prima pasa por varios procesos operativos en los cuales un equipo multidisciplinario se encarga de construir cada uno de los componentes requeridos en el modelo. Los macro procesos identificados en la fabricación son: rayado, corte, taladrado, dobla- do, laminado en fibra de carbono, proceso de mecanizado CNC, torneado, soldado, pulido, pintura y ensamblaje. Estos macro procesos a su vez poseen tareas que se han detallado en hojas de trabajo y diagramas de procesos que son entregadas a los operarios por parte del supervisor de producción antes de realizar el trabajo.

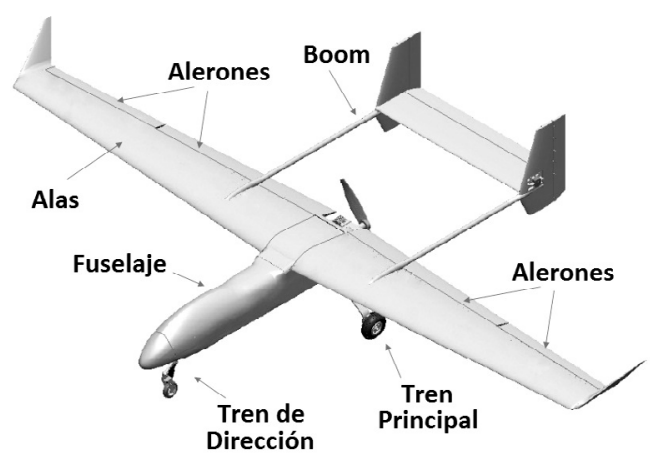

Fig.2. Sub-ensambles principales del UAV Gavilán III Fuente: Autores.

En el área de producción existen varios talleres con maquinaria y mobiliario especializado para las actividades a desarrollar. Éstos trabajan como células de trabajo. Para facilitar las tareas de producción, se asignó un código a cada uno de los componentes y, a su vez, se distribuyó la producción de acuerdo con el material a mecanizar y componentes a fabricar.
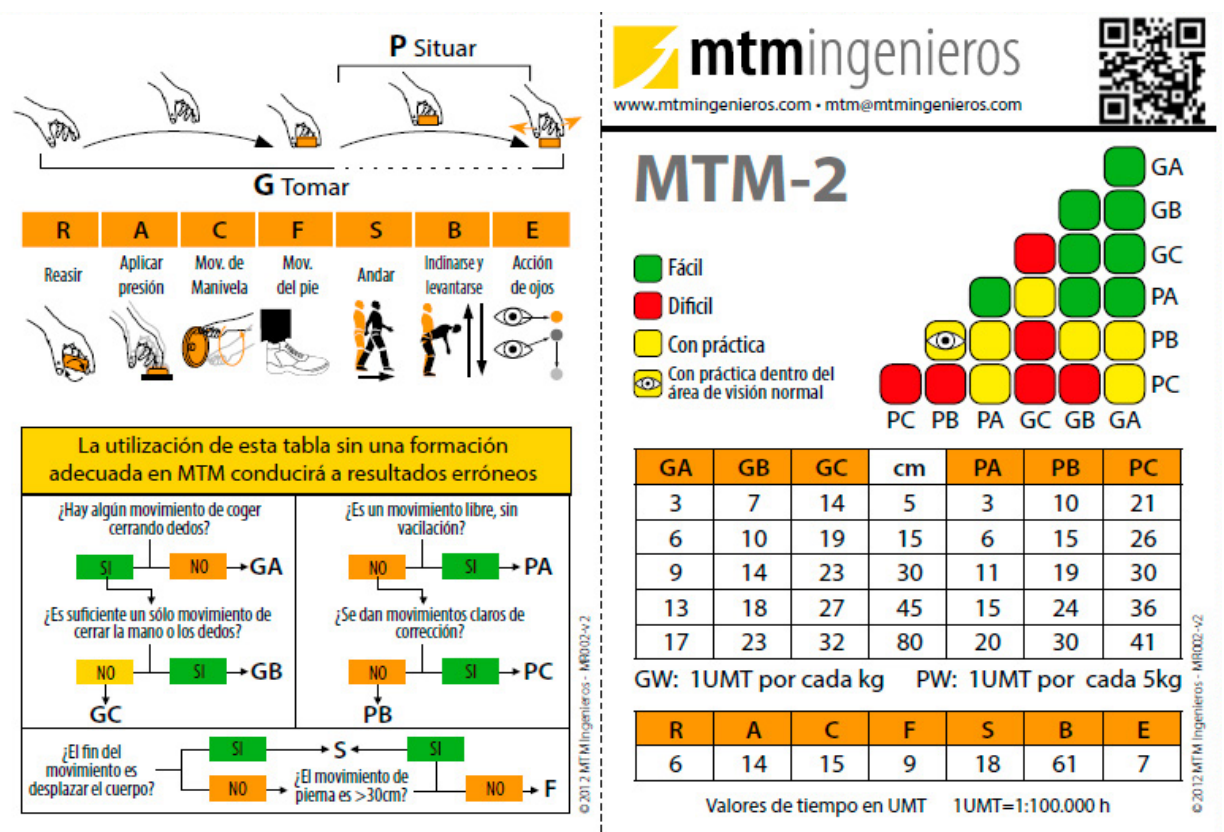

Fig. 3. Tabla de tiempos predeterminados MTM-2. Fuente: [25]. 


\section{E. Estudio de tiempos con base en el sistema MTM-2}

Para el uso de la normativa MTM-2 se deben considerar los micromovimientos básicos, denominados therbligs, mostrados en el costado izquierdo de la Fig. 3 y utilizando herramientas diagramas hombremáquina. A cada movimiento se le asigna una valoración que viene dada en TMU, posteriormente se realiza la conversión de unidades correspondiente de acuerdo con lo indicado en [12]. Para describir la aplicación de este sistema, se expone el ejemplo descrito a continuación, que corresponde al proceso de fijación de la base del tren de dirección del UAV Gavilán III (Fig. 4).

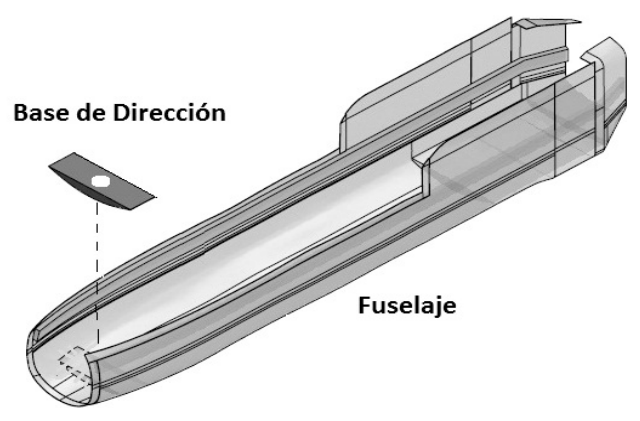

Fig. 4. Fijación de la base del tren de dirección dentro del fuselaje. Fuente: Autores.

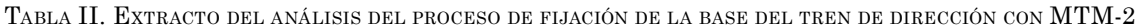

\begin{tabular}{|c|c|c|c|c|c|c|c|}
\hline Tareas Mano Izquierda & MI & TMU & $\begin{array}{c}\text { Tiempo } \\
\text { Normal } \\
\text { (TMU) }\end{array}$ & TMU & & MD & Tareas Mano Derecha \\
\hline \multirow{2}{*}{ Tomar piel } & GB30 & 14 & 23 & 23 & & GB80 & \multirow{2}{*}{ Tomar piel } \\
\hline & $3 \mathrm{GW}$ & 1 & 3 & 1 & 3 & GW & \\
\hline \multirow{5}{*}{ Situar piel } & PB45 & 24 & 30 & 30 & & PB80 & \multirow{2}{*}{ Situar piel } \\
\hline & PW5 & 1 & 1 & 1 & & PW5 & \\
\hline & & 0 & 7 & 7 & & $\mathrm{E}$ & Visualizar posición de lija \\
\hline & & 0 & 27 & 27 & & GC45 & Tomar lija \\
\hline & & 0 & 15 & 15 & & PA45 & Situar lija \\
\hline \multirow[t]{5}{*}{ Sostener piel de fuselaje } & GB10 & 10 & 360 & 15 & 24 & PA45 & Lijar superficie \\
\hline & & 0 & 15 & 15 & & PA45 & Situar lija en reposo \\
\hline & & 0 & 7 & 7 & & $\mathrm{E}$ & Visualizar paño \\
\hline & & 0 & 23 & 23 & & GB50 & Tomar paño \\
\hline & & 0 & 20 & 20 & & PA50 & Situar paño \\
\hline \multirow[t]{5}{*}{ Sostener piel de fuselaje } & GB10 & 10 & 90 & 15 & 6 & PA45 & Limpiar superficie \\
\hline & & 0 & 20 & 20 & & PA50 & Situar paño en reposo \\
\hline & & 0 & 7 & 7 & & $\mathrm{E}$ & Visualizar pegamento \\
\hline & & 0 & 23 & 23 & & GB80 & Tomar pegamento \\
\hline & & 0 & 30 & 30 & & PB80 & Situar pegamento \\
\hline Situar pegamento & GB15 & 10 & 23 & 23 & & GB70 & Tomar brocha \\
\hline \multirow[t]{2}{*}{ Sostener piel de fuselaje } & GB10 & 10 & 88 & 11 & 8 & PA30 & Aplicar pegamento \\
\hline & & 0 & 30 & 30 & & PB80 & Situar pegamento en reposo \\
\hline Visualizar base & $\mathrm{E}$ & 7 & 7 & 0 & & & \\
\hline Alcanzar base & $2 \mathrm{~S}$ & 18 & 36 & 0 & & & \\
\hline Tomar base & GB80 & 23 & 23 & 0 & & & \\
\hline Situar base & PA80 & 20 & 20 & 0 & & & \\
\hline Situar base & $\mathrm{PC} 45$ & 36 & 36 & 36 & & $\mathrm{PC} 45$ & Situar base \\
\hline Aplicar presión & $\mathrm{A}$ & 14 & 14 & 14 & & A & Aplicar presión \\
\hline Situar mano en reposo & PB45 & 24 & 24 & 24 & & PB45 & Situar mano en reposo \\
\hline
\end{tabular}


La Tabla II corresponde a un extracto de varias operaciones realizadas durante el proceso de ensamble del fuselaje del avión. Para efectuar este proceso, el operario toma y sitúa el revestimiento del fuselaje fabricado en fibra de carbono sobre la mesa de trabajo (este componente tiene un peso aproximado de $5 \mathrm{~kg}$ ); sujeta el componente con una mano mientras que con la otra lija la superficie en la cual se fijará el componente secundario; luego se limpia con un paño la superficie para eliminar los residuos; se aplica pegamento en las superficies y se fija el componente secundario, que en este caso es la base de dirección en la posición establecida; y de este modo, el operario concluye la actividad.

Al analizar la primera tarea del ejemplo en la Tabla II, que es "tomar piel", se tienen en cuenta dos aspectos importantes: el recubrimiento o piel de fuselaje de acuerdo con su diseño, el cual es un elemento que se puede tomar fácilmente con sólo cerrar las manos, por lo cual se le asigna el código GB; y la distancia a la cual debe manipular el elemento, que es de $30 \mathrm{~cm}$ para la mano izquierda (GB30) y de $60 \mathrm{~cm}$ para la mano derecha; de acuerdo con la Fig. 5, para este último valor se toma el código (GB80) ya que abarca las distancias comprendidas entre 46 y $80 \mathrm{~cm}$ de longitud. De acuerdo con la tabla MTM-2 de la Fig.5 se tiene: para GB30 = 14 TMU (mano izquierda) y para GB80 $=23$ TMU (mano derecha). De estos dos valores mencionados, se toma 23 TMU debido a que es el tiempo que restringe la actividad. El revestimiento o piel de fuselaje tiene un peso aproximado de $5 \mathrm{~kg}$ y se le asignan las siglas GW. Como el operario utiliza sus dos manos para agarrar el objeto, éste tiene un equivalente de $3 \mathrm{~kg}$ de peso por cada mano, de este modo se adopta la codificación $3 \mathrm{GW}$. Según la MTM-2, se debe asignar 1 TMU por cada kg de peso, por lo tanto: $3 \mathrm{GW}=3 \mathrm{TMU}$ (para cada mano). Para el tiempo normal, se toma un valor promedio equivalente a 3 TMU.

Para cada una de las demás tareas, el proceso se realiza de manera similar ubicando los códigos correspondientes a cada actividad con su respectivo valor en TMU. Para obtener el tiempo normal total de la operación, se suman todos los valores de tiempo normal en TMU de la columna. Entonces, para el proceso de fijación de la base del tren se tiene un valor total de 1002 TMU. Finalmente, este valor de tiempo necesita ser transformado a unidades de tiempo fácilmente identificables, para eso se tiene en cuenta la siguiente equivalencia: $1 \mathrm{TMU}=0.036$ seg [25]. Mediante esta consideración, se tiene que el tiempo normal para realizar la fijación de la base del tren de dirección equivale a 36,07 segundos. Una vez obtenidos todos los tiempos de las operaciones requeridas para la fabricación de piezas se crean matrices de resumen de tiempos para cada sub-ensamble principal, como el que se indica en la Tabla III.

TABla III. Matriz de Resumen de tiempos para ELABORACIÓN DE PIEZAS QUE CONFORMAN LOS FLAPS

\begin{tabular}{|cc|cc|c|}
\hline \multicolumn{4}{|c|}{ Tiempo estimado de fabricación de piezas de los flaps (min.) } \\
\hline Componente & Laminado & $\begin{array}{c}\text { Mec. CNC } \\
\text { TAM TCM }\end{array}$ & Pulido \\
\hline Costillas 1 & 21 & 32 & 42 & 8,7 \\
Costillas 2 & - & 32 & 12 & - \\
Cuerpo principal & - & 32 & 38 & 2,6 \\
Piel flap vertical & 36 & & - & 5,6 \\
Soporte de dirección & 23 & & - & - \\
\hline \multicolumn{2}{|c|}{ T. Actividades Manuales } & 96 & \\
\hline
\end{tabular}

Fuente: Autores

En ciertos casos, como el del proceso de mecanizado CNC, se incluyen dos tiempos: tiempos de las actividades manuales desarrolladas por el operario (TAM) y el tiempo condicionado por la máquina (TCM) al realizar su trabajo.

Para la evaluación del trabajador se asigna una valoración de $100 \%$, ya que los operarios poseen la destreza necesaria para realizar su trabajo. Los suplementos por descanso a las actividades desarrolladas por los operarios, fueron establecidos con base en un análisis de las condiciones del lugar de trabajo y las operaciones que desempeñan los trabajadores de la sección. Se consideraron factores de tensión física, tensión mental y las condiciones de trabajo, y se asignó una calificación de acuerdo con los puntos atribuidos a las diferentes tensiones especificados por la OIT [12], [32]. En la Tabla IV se sintetizan los tiempos de operación estimados para cada proceso principal y sus respectivos suplementos de descanso.

Tabla IV. Cálculo del tiempo estándar total y PARCIAL PARA LA PRODUCCIÓN DE UAV GAVILÁN III

\begin{tabular}{|c|c|c|c|c|c|}
\hline \multicolumn{2}{|c|}{$\begin{array}{l}\text { Producto: } \\
\text { UAV Gavilán III }\end{array}$} & \multirow{2}{*}{$\begin{array}{c}\text { Tiempo } \\
\text { básico } \\
\text { (hrs) }\end{array}$} & \multirow[t]{2}{*}{$\begin{array}{l}\text { Descanso } \\
(\%)\end{array}$} & \multirow[t]{2}{*}{$\begin{array}{c}\text { Suplemento } \\
\text { (h) }\end{array}$} & \multirow{2}{*}{$\begin{array}{c}\text { Tiempo } \\
\text { estándar } \\
\text { (h) }\end{array}$} \\
\hline $\mathrm{N}^{\circ}$ & Operaciones & & & & \\
\hline A & Rayado & 1,550 & 26,00 & 0,40 & 1,96 \\
\hline $\mathrm{B}$ & Cortar & 3,380 & 39,00 & 1,32 & 4,70 \\
\hline $\mathrm{C}$ & Taladrar & 0,260 & 35,00 & 0,09 & 0,35 \\
\hline $\mathrm{D}$ & Doblar & 0,730 & 32,00 & 0,23 & 0,96 \\
\hline $\mathrm{E}$ & Mec. TAM & 18,130 & 5,44 & 56,22 & \\
\hline E & CNC TCM & 32,650 & & & 55,32 \\
\hline $\mathrm{F}$ & Laminado & 16,530 & 35,00 & 5,79 & 22,32 \\
\hline G & Tornear & $\begin{array}{l}0,180 \\
0,600\end{array}$ & 0,06 & 0,84 & 0,83 \\
\hline $\mathrm{H}$ & Soldado & 0,550 & 27,00 & 0,15 & 0,70 \\
\hline I & Pulir & 2,810 & 39,00 & 1,10 & 3,91 \\
\hline $\mathrm{J}$ & Pintar & 7,100 & 48,00 & 3,41 & 10,51 \\
\hline \multirow[t]{2}{*}{$\mathrm{K}$} & Ensamblar & 2,640 & 30,00 & 0,79 & 3,43 \\
\hline & & \multicolumn{3}{|c|}{ Tiempo estándar total: } & $105,89 \mathrm{~h} / \mathrm{u}$ \\
\hline
\end{tabular}


Estimado el tiempo estándar, es posible calcular la capacidad de producción basados en (1):

$$
C P=\frac{1}{T s}
$$

Donde $C P$ representa la capacidad de producción y Ts el tiempo estándar. Para el cálculo se debe tener en cuenta que los operarios trabajan jornadas de 8 horas diarias por 20 días laborables al mes.

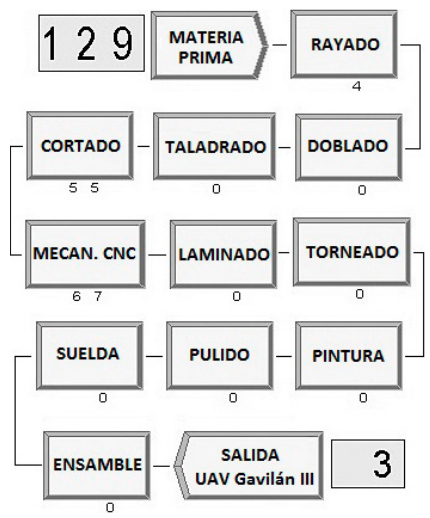

Fig. 5. Simulación del modelo con 320 horas laborables. Fuente: Autores.

En la Fig. 5, se presenta el resultado de la simulación realizada con el software Arena para un tiempo de producción de 320 horas laborables, es decir, dos meses de trabajo. La interfaz cuenta con un área gráfica en la cual se desarrolla el modelo a partir de figuras geométricas que corresponden a las entradas, procesos y salidas que se requieren para la construcción del modelo. En este periodo de tiempo se observa que el programa arroja un resultado de 3 unidades UAV terminadas a su salida.

\section{Fase III: Análisis de las instalaciones y distribución de planta}

En lo referente al ensamble final del UAV, se realiza un balance de la línea de ensamble. Debido a la gran cantidad de tareas a realizar se utilizó el módulo Facility Location and Layout perteneciente al software WinQSB. Se determina idóneo dividir el área de ensamble en cuatro estaciones de trabajo cuyo tiempo de ciclo es de 49,37 minutos, y dependiendo de las tareas que se han asignado a cada estación de ensamble se elaboró una lista de los componentes requeridos en cada una de las mismas. Las actividades que se asignaron a cada estación son: estación de trabajo 1, en la cual se realiza el montaje del fuselaje y trenes de aterrizaje con el propósito de facilitar el transporte del sub-ensamble de una estación de trabajo a la siguiente; estación 2 , en la cual se realiza el montaje de los estabilizadores de la cola del avión, alerones y flaps; estación 3, en esta estación de trabajo se realiza el montaje final de las alas y el ensamblaje final de la cola del avión, más conocida como boom; y estación 4 , donde se realiza el montaje final de las alas y la cola del avión al fuselaje en su posición definitiva. Una vez culminados los procesos de montaje, la estructura del avión pasa a otra sección del CIDFAE encargada de la parte electrónica en la cual se le añaden al avión los componentes y sistemas electrónicos de control, navegación, energía, etc., y finalmente, se pasa a las respectivas pruebas y calibración de la aeronave.

Para determinar el espacio requerido por las instalaciones se utiliza el método de Guerchet, el cual evalúa el espacio físico con base en tres parámetros: la superficie estática (Ses), que es la superficie productiva que ocupa físicamente la maquinaria, el mobiliario, y demás; la superficie de gravitación (Sg), que es la superficie utilizada por los operarios que están laborando y la materia prima que se está procesando, para su cálculo se utiliza (2); y la superficie de evolución (Sev), que es la superficie necesaria para el movimiento del personal, materiales y sus medios de transporte, viene dada (3). El número de lados operativos de la máquina está representado por $n$. La superficie total (ST), viene dada por la sumatoria de las superficies mencionadas anteriormente, como se observa en (4).

$$
\begin{aligned}
& S g=S e s * n \\
& S e v=(S e s+S g) * k \\
& S T=S e s+S g+S e v
\end{aligned}
$$

El coeficiente de proporción (k) entre el volumen del material y el personal que se está moviendo entre los puestos de trabajo. Se utiliza la valoración correspondiente a este tipo de industria [29]. El cálculo del espacio total requerido se muestra en la en la Tabla V.

Tabla V. Superficie total Requerida para LOS TALLERES DE LA SECCIÓN DE PRODUCCIÓN

\begin{tabular}{|l|c|}
\hline \multicolumn{2}{|c|}{ Superficie Requerida } \\
\hline Taller & Area requerida $\mathbf{( m}^{\mathbf{2}} \mathbf{~}$ \\
Mecánica & 71,41 \\
Materiales Compuestos & 43,96 \\
Carpintería & 41,38 \\
Ensamblaje & 1032,64 \\
Pintura & 44,64 \\
\hline Superficie total: & $\mathbf{1 2 3 4 , 0 3} \mathbf{~ m}^{\mathbf{2}}$ \\
\hline
\end{tabular}

Fuente: Autor

El costo de transporte de material dentro de cada uno de los talleres se evaluó mediante el software WinQSB. Debido a que cada taller posee maquinaria para fabricar los distintos componentes, se diseña la distribución inicial basada en los procesos de la instalación, como se muestra en la Fig. 6. Los datos obtenidos se ingresan en el software para obtener la mejor solución con el menor costo de transporte de materiales. 


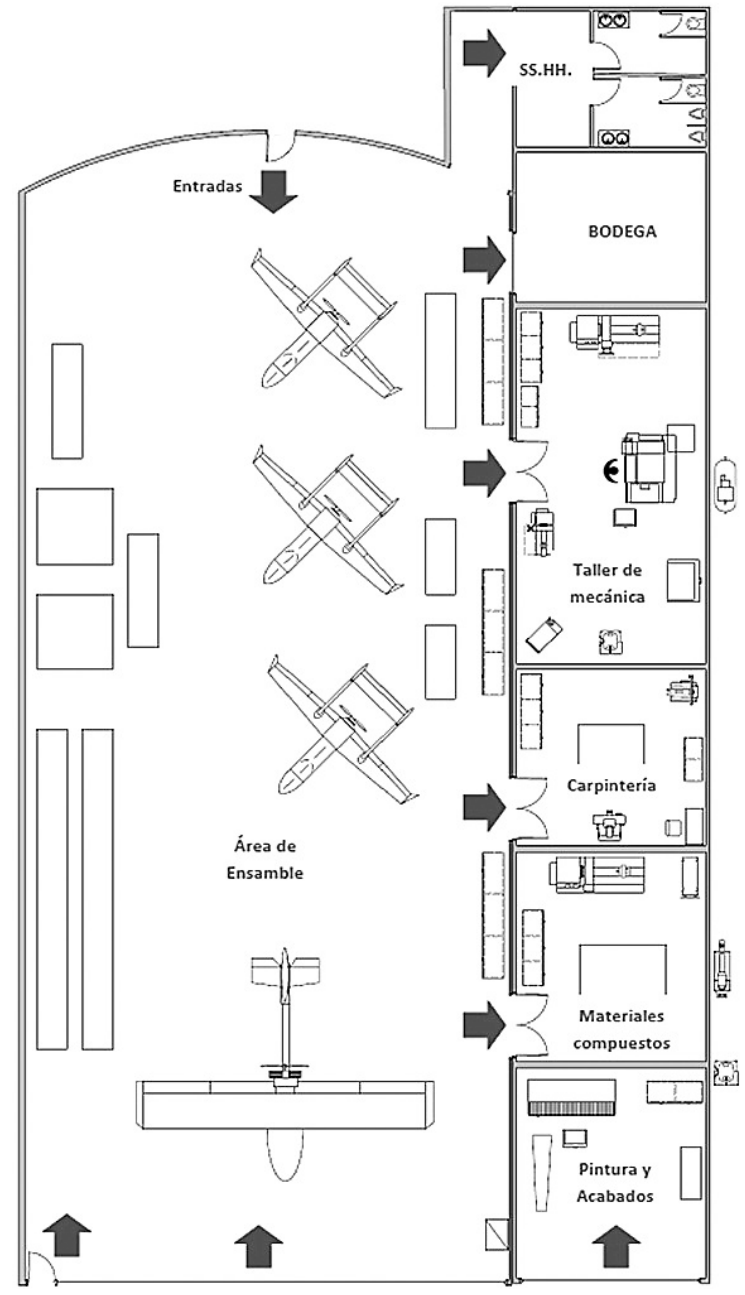

Fig. 6. Distribución actual de la planta de la sección de producción. Fuente: Autor.

Como resultado del análisis con el software WinQSB, se tiene que el tipo de distribución final para la sección de producción es una distribución mixta, en la cual los distintos talleres son las células de trabajo dentro las cuales se adoptó una distribución por proceso. Para el ensamble final se trabaja como una distribución por posición fija debido a las características físicas del aeroplano, este espacio de trabajo se divide en 4 estaciones de acuerdo a lo mencionado anteriormente en el balanceo de la línea de ensamble. La distribución propuesta presenta una reducción de la distancia entre puestos de trabajo optimizando el flujo de materiales, lo cual implica una reducción por costos de transporte de los mismos. En la Fig. 7 se presenta la distribución mixta final propuesta obtenida para la sección de producción y ensamble. La sección del ensamble final se divide en 4 estaciones de acuerdo con lo mencionado anteriormente en el balanceo de la línea de ensamble.

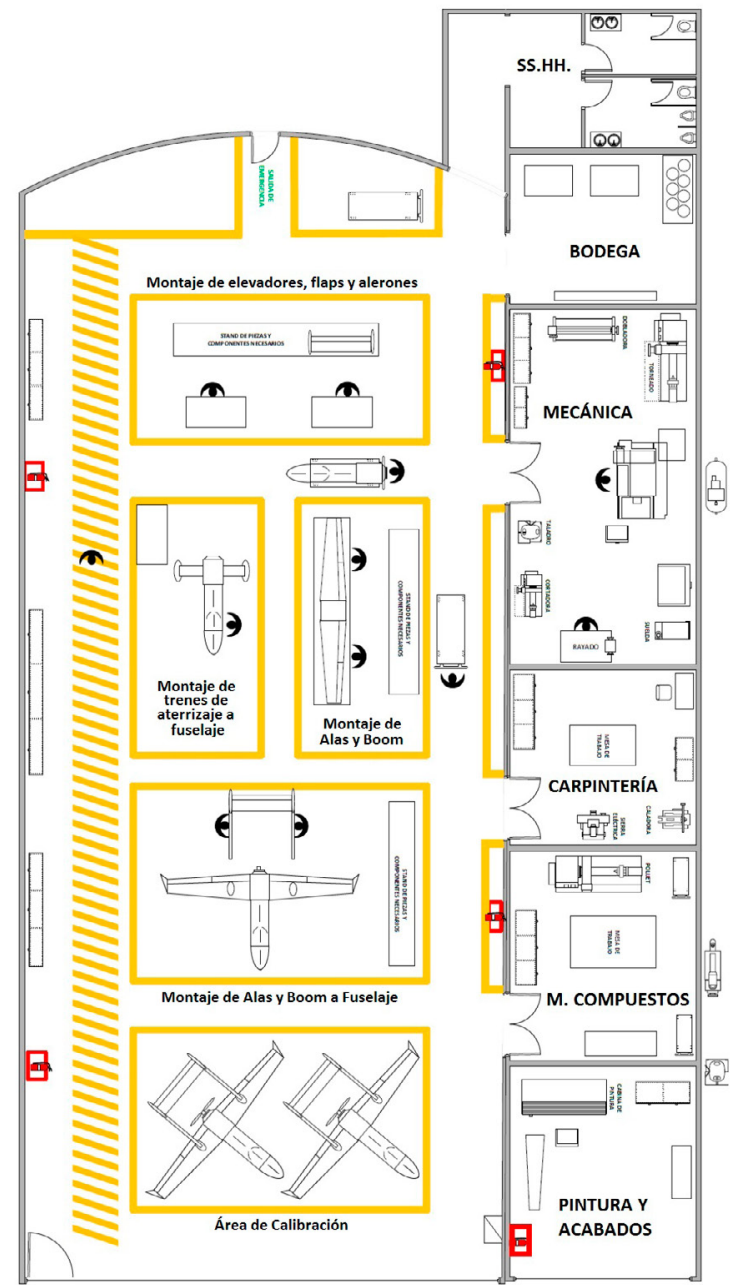

Fig. 7. Distribución de planta propuesta para la sección de producción. Fuente: Autores.

Finalmente, para una distribución óptima de los puestos de trabajo es importante que las instalaciones cumplan con las normativas de seguridad laboral vigentes en el país, para ello se utiliza el Decreto ejecutivo 2393 que es el Reglamento de salud y seguridad de los trabajadores, en el cual se establecen una serie de parámetros a cumplir referentes a instalaciones, equipos de protección personal, extintores, etc... [30]. En lo referente a señalización de seguridad, se adoptan los requerimientos establecidos en la Norma Técnica Ecuatoriana NTE INEN ISO 3864-1:2013 [31].

\section{Discusión}

De acuerdo con el análisis de la situación estratégica actual y el análisis del sector industrial, se identifica que existe un alto grado de competencia a nivel de Latinoamérica, siendo Argentina y Brasil los países que lideran este tipo de industria. 
Para que el CIDFAE pueda ingresar en este mercado, y a su vez pueda promocionar el producto tanto a nivel nacional como a otros países de Latinoamérica, primeramente debe adoptar las estrategias de producción planteadas, las cuales le permitirán mejorar la calidad de los procesos y, por ende, la calidad del producto final. Entre estas estrategias están la adquisición de maquinaria de última generación, la aplicación de planes de mantenimiento preventivo para maquinaria y equipos, y otros ya establecidos. Con base en este análisis se considera que el CIDFAE no se encuentra en las condiciones apropiadas para efectuar la producción en serie del modelo Gavilán III.

Como resultado del análisis del proceso productivo, se identificaron los componentes requeridos para el ensamblaje del UAV Gavilán III. Al existir una gran cantidad de componentes, es necesario establecer una codificación para tener un mejor control de los mismos. Asimismo, con fundamento en el análisis de tiempos realizado mediante la Normas MTM-2 para la fabricación de los distintos componentes se han identificado procesos que limitan la producción actual a tres aviones por cada dos meses de trabajo. El proceso de mecanizado $\mathrm{CNC}$ requiere tiempos elevados para preparación, carga y descarga de la máquina; estos tiempos se pueden reducir mediante un entrenamiento adecuado del personal a cargo de la maquinaria. Además, se propone la creación de plantillas para mecanizado de varios componentes que requieran del mismo material. Como una alternativa adicional se puede optar por la adquisición de una nueva máquina CNC de similares características que permita reducir el cuello de botella que se forma debido a la gran cantidad de componentes que requieren de este proceso para su fabricación.

Otro de los procesos que representan un cuello de botella es el proceso de laminado, pues éste demanda preparación y tratamiento adecuado de los materiales. Para solucionar este inconveniente se requiere de la elaboración de un juego de moldes adicional para la creación del revestimiento de los distintos sub-ensambles principales. Por el contrario, para el proceso de corte se considera necesaria la reactivación de la máquina caladora existente, realizando previamente un chequeo y reparación de la misma, ya que este proceso es uno de los más utilizados en la fabricación de los distintos componentes.

En lo referente a infraestructura y mediante el análisis realizado con el software WinQSB, la sección de producción cuenta con el espacio necesario para reubicar la maquinaria, equipos y mobiliario de manera eficiente. Se consiguió establecer una propuesta de distribución eficiente para reducir las distancias y costos por transporte de materiales entre puestos de trabajo, teniendo como resultado una distribución mixta en la cual se tiene una distribución por proceso dentro de los diferentes talleres.

El UAV Gavilán III ha sido diseñado para operaciones de grandes alturas en los andes ecuatorianos, por lo que las instalaciones de su estación de control en tierra y pista de aterrizaje están ubicadas en la región central del país en la sierra, específicamente en la ciudad de Ambato. Estas condiciones presentan un escenario ideal para sus operaciones aéreas.

\section{Conclusiones}

Se puede constatar que tanto la planeación de producción y la administración estratégica constituyen las tareas de gran importancia para la alta gerencia, por esta razón, estos elementos requieren de innovación continua para asegurar el éxito de la organización.

Gracias al análisis del sector industrial, se identifica que la CIDFAE no se encuentra en capacidad de competir con fabricantes de UAV a nivel de Latinoamérica. Existen varias empresas que lideran el mercado como Tesacom, Volartec, Aerodreams. El CIDFAE, por su lado, para asegurar su posicionamiento en el mercado establece planes de acción y estrategias para mejorar la calidad de los productos, y mediante la aplicación de éstas plantea reducir el desperdicio de materiales ocasionado por una mala organización de la producción y, a su vez, explotar al máximo la capacidad operativa de la institución.

En cuanto al estudio del proceso productivo, se vio la necesidad de realizar un estudio mediante estimaciones de tiempos utilizando herramientas como diagramas hombre-máquina, normas de tiempo predeterminadas MTM-2 y la colaboración de los operarios con base en su experiencia laboral. De este análisis, se obtiene como resultado que el CIDFAE actualmente está en capacidad de producir tres aviones cada dos meses de trabajo.

La fabricación del modelo UAV Gavilán III requiere de un alto tiempo de proceso, sin embargo, es muy útil conocer al detalle cada una de las actividades que se desarrollan con el tiempo requerido para las mismas, puesto que se puede conocer la capacidad de producción instalada y llevar a cabo correcciones en el proceso productivo de manera fácil y rápida. Por otro lado, la creación de familia de partes ayuda en gran medida a organizar la producción, ya que de este modo se logra agrupar todos los componentes que requieren de materiales y procesos de fabricación similares, optimizando los recursos y reduciendo el desperdicio de materiales.

La aplicación del software Arena permitió analizar el escenario de una manera rápida y segura. Teniendo en cuenta las restricciones de capacidad de cada proceso como resultado de la simulación, se obtuvo una producción de tres aviones por cada dos meses de trabajo. Mediante el uso del software WinQSB, se pudieron conseguir múltiples alternativas de solución para la ubicación de maquinaria dentro 
de cada taller, teniendo en cuenta los costos de transporte y flujo de material entre puestos de trabajo. En el análisis de flujo de material de la distribución propuesta se obtuvo un costo de 65 dólares frente a los 86,4 dólares de la distribución actual, así, como resultado final existe un ahorro del $25 \%$ en costo de flujo de material.

Para el diseño final del layout de la sección de producción se toma la información del balance de la línea de ensamblaje y los reglamentos vigentes en el país referentes a seguridad laboral. De este modo, en la Fig. 7 se tiene una distribución mixta en donde cada taller provee los componentes necesarios al área de montaje, la misma que se organiza en cuatro estaciones de trabajo a para efectuar el ensamblaje final de los UAV y que cumple con las normativas de seguridad.

En relación con la estación de control en tierra, actualmente la CIDFAE utiliza las instalaciones del aeropuerto Chachoán de la ciudad de Ambato. El aeropuerto está en una elevación de 2,591 metros, tiene una pista de aterrizaje designada 01/19 con superficie de asfalto que mide 1,925 x 25 metros. Para estudios posteriores se sugiere realizar un detallado análisis de la estación de control en tierra.

\section{Agradecimientos}

Los autores agradecen al Ministerio de Defensa Nacional, la Fuerza Aérea Ecuatoriana y la Universidad Técnica de Ambato por el apoyo brindado para la realización de la investigación en el marco de ejecución del proyecto de titulación de Ingeniería Industrial en Procesos de Automatización denominado "Procesos de ensamblaje de aviones no tripulados para el Centro de Investigación y Desarrollo de la Fuerza Aérea Ecuatoriana (CIDFAE)".

\section{REFERENCIAS}

[1] D. Raymer, "Enhancing Aircraft Conceptual Design using Multidisciplinary Optimization,” Institutionen för flygteknik, Tesis de doctorado, Dept. Aeron., Royal Instit. of Technology., Stockholm, Sweden, 2002.

[2] G. Sánchez Jiménez, M. Mulero Valenzuela, and E. Saumeth Cadavid, "Vehículos aéreos no tripulados en Latinoamérica," idsolution, Madrid, pp. 8-85, May-2013.

[3] V. Enríquez, "Del proceso de investigación y desarrollo al escalamiento industrial," WordPress.com, 2014. [En línea]. Disponible en: https://vxenriquezblog.wordpress. com/2014/09/29/del-proceso-de-investigacion-y-desarrollo-al-escalamiento-industrial/.

[4] C. Williams, Management, 6th ed. South-Western: Cengage Learning, 2011.pp. 828.

[5] S. C. Certo and J. P. Peter, Dirección estratégica, 3rd ed. Madrid: McGraw-Hill Interamericana, 1997. pp.382

[6] S. Hart and C. Banbury, "How strategy-making processes can make a difference," Strateg. Manag. J., vol. 15, no. 4, pp. 251-269, may 1994. DOI: $10.1002 /$ smj.4250150402

[7] F. V. Ospino Valdiris, "El direccionamiento estratégico basado en las opiniones de los empleados: estrategia para lograr las metas," INGE CUC, vol. 6, no. 1, pp. 167-180, oct. 2010.
[8] L. S. Cárdenas, E. B. Ibarra, and B. A. García, "Una metodología innovadora para la planeación estratégica en las universidades," INGE CUC, vol. 9, no. 1, pp. 43-63, jul. 2013.

[9] N. Gaither and G. Frazier, "Disposición física de instalaciones," en Administración de producción y operaciones, 8th ed., Cengage Learning Latin Am, 2000, pp. 43,268-269.

[10] N. C. Solano and R. Á. P. Murgía, "Optimización del proceso de fabricación de alambres de aleación 6201 T-81," INGE CUC, vol. 7, no. 1, pp. 207-216, oct. 2011.

[11] A. Freivalds and B. W. Niebel, "Análisis de operaciones," en Ingeniería industrial: métodos, estándares y diseño del trabajo, 11th ed., México: McGraw-Hill, 2009.

[12] G. Kanawaty, "Disposición del espacio, manipulación y planificación del proceso," en Introducción al estudio del trabajo, 4th ed., Ginebra: Oficina Internacional del Trabajo, 1996, pp. 207, 387, 410.

[13] R. García Criollo, "Distribución de Planta," en Estudio del Trabajo. Ingenieria de Metodos y Medición del Trabajo, 2nd ed., México: Mc Graw Hill, 2005, pp. 143-145.

[14] P. A. Aguaysa Carrillo and C. J. Mariño Rivera, "Distribución de Planta y su Influencia en el Proceso de Producción del Área de Manufactura en la Empresa Tenería 'Inca' S.A de la ciudad de Ambato", Tesis de maestría, Dept. Electron., Sist. e Ind. Eng., Téc. Ambato, Ambato, Ecuador, 2013.

[15] M. Shouman, G. Nawara, A. Reyad, and K. El-Darandaly, "Facility Layout Problem (FLP) amd Intelligent Techniques: A Survey," 2011.

[16] E. P. López Morales, "Distribución de planta para la optimización del manejo de materiales en la empresa de calzado DAV-SPORT de la Ciudad de Ambato,". M.S. thesis, Dept. Electron., Sist. e Ind. Eng., Téc. Ambato, Ambato, Ecuador, 2014.

[17] R. Sharma and L. R. Foulds, "A Simulation Model of Horseshoe Layouts," en 33rd Annual Conference of the Operational Research Society of New Zealand, 1998.

[18] J. E. González and E. M. Collante, "Análisis de la capacidad del proceso de cargue y descargue en la sociedad portuaria regional Barranquilla SPRB," INGE CUC, vol. 7, no. 1 , pp. $17-26$, oct. 2011.

[19] R. A. G. Montoya, J. A. S. Alzate, and J. A. P. Muñoz, "Análisis de la operación despacho en un centro de distribución basado en gestión de procesos y simulación," INGE CUC, vol. 7 , no. 1, pp. $75-86$, oct. 2011.

[20] E. A. Pacheco Velásquez, "Un modelo para la optimización de políticas de inventario conjuntas en cadenas de suministro," INGE CUC, vol. 9, no. 1, pp. 11-23, jul. 2013.

[21] J. Reyes, K. Alvarez, and R. Vasquez, "Dynamic Buffer Management for Raw Material Supply in the Footwear Industry," J. Ind. Intell. Inf., vol. 4, no. 1, pp. 1-8, 2016. DOI: 10.12720/jiii.4.1.1-8

[22] J. P. Reyes Vasquez and C. G. Molina Velis, "Plan agregado de producción mediante el uso de un algoritmo de programación lineal: Un caso de estudio," Rev. Politécnica, vol. 34, no. 1, ago. 2014.

[23] J. C. Cabarcas Reyes, M. J. Wilches-Arango, A. F. Chaves, and S. M. Sanmiguel, "Análisis y mejoramiento de la cadena de valor de la línea de producción de láminas de una empresa del sector metalmecánico mediante la aplicación de herramientas de manufactura Lean," INGE CUC, vol. 7, no. 1 , pp. $27-42$, oct. 2011.

[24] L. Goodstein, T. Nolan, and J. Pfeiffer, Applied Strategic Planning: How to Develop a Plan That Really Works, 3rd ed. Estados Unidos: Mc Graw Hill, 1993.

[25] MTM Ingenieros, “QQué es el MTM?” [En línea]. Disponible en: http://mtmingenieros.com/knowledge/que-es-el$\mathrm{mtm} /$.

[26] N. Alzate Guzmán and J. E. Sánchez Castaño, "Estudio de métodos y tiempos de la línea de producción de calzado tipo 'clásico de dama' en la empresa de calzado Caprichosa para definir un nuevo método de producción y determinar el tiempo estándar de fabricación,” Tesis de maestría, Dept. Ind. Eng., Univ. Tecn. Pereira, Pereira, 2013. 
CONSIDERACIONES PARA EL REDISEÑO DE LA LÍNEA DE ENSAMBLE DE VEHÍCULOS AÉREOS NO TRIPULADOS DESARROLLADOS POR LA FUERZA AÉREA ECUATORIANA

[27] A. C. Hax, "El Modelo Delta un nuevo marco estraté gico," J. Strateg. Manag. Educ., 2003.

[28] M. Baca Cisneros, "Como se diseña un UAV," AeroMundo Magazine, 2013.

[29] L. C. Arbós, Organización de la producción y dirección de operaciones: Sistemas actuales de gestión eficiente y competitiva. Madrid: Ediciones Díaz de Santos, 2012 .
[30] Instituto Ecuatoriano de Seguridad Social, Reglamento de Seguridad y Salud de los Trabajadores y Mejoramiento del Medio Ambiente de Trabajo. Ecuador, 2012.

[31] Instituto Ecuatoriano de Normalización, Norma Técnica Ecuatoriana NTE INEN-ISO 3864-1:2013. Ecuador, 2011.

[32] J. P. Reyes Vásquez, "Distribución de Instalaciones," en Estudio del Trabajo Aplicaciones en la Industria Ecuatoriana, 1st ed., Ambato, 2014, pp. 56-57. 